\title{
Effects of additive noise on vibrational resonance in a bistable system
}

\author{
J. Casado-Pascual* \\ Universidad de Sevilla, Física Teórica, Apartado de Correos 1065, Sevilla 41080, Spain \\ J. P. Baltanás ${ }^{\dagger}$ \\ Departamento de Matemáticas y Física Aplicadas y Ciencias de la Naturaleza, Universidad Rey Juan Carlos, \\ Tulipán s/n, Móstoles 28933, Madrid, Spain
}

(Received 16 September 2003; published 26 April 2004; publisher error corrected 12 May 2004)

\begin{abstract}
We study the overdamped motion of a particle in a bistable potential subject to the action of a bichromatic force and additive noise, within the context of the vibrational resonance phenomenon. Under appropriate conditions, we obtain analytical expressions for the relevant observables which quantifies this phenomenon. The theoretical results are compared with those obtained by the numerical solution of the stochastic differential equation which describes the dynamics of the system. The limits of validity of the theoretical approach are also discussed.
\end{abstract}

DOI: 10.1103/PhysRevE.69.046108

PACS number(s): 05.90. $+\mathrm{m}, 05.40 .-\mathrm{a}, 05.45 .-\mathrm{a}$

\section{INTRODUCTION}

During the last three decades, a large amount of work has been devoted to the study of nonlinear systems subject to noise and time-dependent forces. In the course of these studies it has become clear that, in contrast to the role played by stochastic forces in linear systems, noise can drastically alter the response of nonlinear dynamical systems to the external forcing under some particular circumstances. A particularly interesting example of the effects of noise within the framework of signal processing by nonlinear systems is stochastic resonance (SR), i.e., the amplification of a weak input signal by the concerted actions of noise and the nonlinearity of the system. Although discussed initially within the context of dynamical systems with bistable potentials [1], the phenomenon of SR has also been found in other dynamical systems [2,3], including systems of biological interest (see, e.g., Ref. [4] and references therein). Many authors have studied the occurrence of SR not only in the processing of harmonic signals but also of aperiodic signals [5-9], and in the presence of both white and colored noise [10-13]. Furthermore, similar effects have been also found when a chaotic signal is used instead of noise [14,15].

The paradigmatic model in the study of SR is the overdamped motion of a particle in a symmetric double-well potential driven by a periodic signal and noise. Its dynamics is described by the stochastic differential equation (SDE)

$$
\dot{x}(t)=-U^{\prime}[x(t)]+F(t)+\xi(t),
$$

where $U^{\prime}(x)$ is the derivative with respect to $x$ of the symmetric double-well potential $U(x), F(t)$ represents the periodic forcing, and $\xi(t)$ is a zero-average Gaussian white noise with autocorrelation function $\langle\xi(t) \xi(s)\rangle=2 D \delta(t-s)$. SR in this model can be understood in terms of the synchronization

\footnotetext{
*Electronic address: jcasado@us.es

†Electronic address: baltanas@escet.urjc.es
}

of two time scales: the Kramers time, characterizing the noise-induced interwell transitions, and the time scale associated to the external driving. Roughly speaking, when Kramers' time matches half the period of the external driving for a given noise strength $D$, the amplification of the weak signal $F(t)$ is optimal.

Recently, an analogous phenomenon named vibrational resonance (VR) has been shown to occur when the noise is replaced by a high-frequency periodic force of varying amplitude. Originally described by Landa and McClintock [16], its study has also been addressed by other authors and from different points of view, in excitable [17], spatially extended [18], and bistable systems [19,20]. The interplay of two monochromatic driving forces has been also considered in the context of controlling stochastic resonance $[21,22]$. In very many physical situations, noise is present and its effects cannot be avoided (for instance, thermal noise or noise arising from the environment). The interplay between noise and nonlinearity can give rise to new aspects of the VR phenomenon, absent in a purely deterministic dynamics. In Ref. [20], a brief numerical study of the effects of additive noise on VR has been presented, but a more detailed investigation of this topic is still lacking. In this paper, we undertake an analytical study of the problem that will provide explicit expressions for the relevant observables. Our analytical results are compared with numerical simulations performed on the model described in Ref. [20].

\section{DESCRIPTION OF THE MODEL AND CHARACTERIZATION OF VR}

We consider a system described by the SDE (1), with $U(x)$ being the symmetric quartic potential in dimensionless form,

$$
U(x)=\frac{x^{4}}{4}-\frac{x^{2}}{2},
$$

and $F(t)$ the bichromatic force 


$$
F(t)=A \cos (\Omega t)+N \Omega r \cos (N \Omega t+\varphi) .
$$

Here, for reasons that will become clear in the following section, we have introduced in the second monochromatic force an arbitrary initial dephasing $\varphi$. The parameter $N$ is chosen to be a positive integer, so that $F(t)$ is periodic with the same period $T=2 \pi / \Omega$ as the first monochromatic force. By $r$ we have denoted the ratio of the amplitude of the second force to its frequency. This ratio $r$ is assumed to be of the same order as the parameters characterizing the potential $U(x)$. We are interested in situations in which the parameters $N \Omega r$ and $N \Omega$ appearing in the second monochromatic force are much larger than the rest of the parameters in the problem. In this sense, we will say that $N \Omega r \cos (N \Omega t+\varphi)$ is a strong, high-frequency monochromatic force. This situation can be formally achieved by taking the limit $N \rightarrow \infty$, with the ratio $r$ kept fixed.

The corresponding Fokker-Planck equation (FPE) for the probability density $P(x, t)$ reads

$$
\frac{\partial}{\partial t} P(x, t)=\frac{\partial}{\partial x}\left[D \frac{\partial}{\partial x}+U^{\prime}(x)-F(t)\right] P(x, t) .
$$

The analysis of this equation is simplified by making use of two important theorems: the $H$ theorem, which ensures the existence of a unique long time distribution function $P_{\infty}(x, t)$ $[23,24]$, and the Floquet theorem, which guarantees that $P_{\infty}(x, t)$ is periodic in time with the same period $T$, as the external force [25]. Henceforth, we will assume that the relaxation transient stage has ended and, consequently, the long time distribution $P_{\infty}(x, t)$ has been reached.

The first moment of the probability distribution can be used to characterize the system response to the external driving. As a consequence of the above mentioned theorems, its long time limit $\langle x(t)\rangle_{\infty}$ is a periodic function of time with period $T$ and, therefore, it can be expanded in Fourier series as follows:

$$
\langle x(t)\rangle_{\infty}=\sum_{n=-\infty}^{\infty} X_{n} e^{i n \Omega t}=X_{0}+2 \sum_{n=1}^{\infty}\left|X_{n}\right| \cos \left(n \Omega t-\phi_{n}\right),
$$

where

$$
X_{n}=\frac{1}{T} \int_{0}^{T} d t\langle x(t)\rangle_{\infty} e^{-i n \Omega t}
$$

and the phases $\phi_{n}$ have been chosen so that

$$
\begin{gathered}
\cos \phi_{n}=\operatorname{Re}\left(\frac{X_{n}}{\left|X_{n}\right|}\right), \\
\sin \phi_{n}=-\operatorname{Im}\left(\frac{X_{n}}{\left|X_{n}\right|}\right) .
\end{gathered}
$$

The observable that has been usually considered to quantify the VR phenomenon is the parameter

$$
Q=2 \frac{\left|X_{1}\right|}{A} .
$$

This parameter represents the ratio of the amplitude associated to the first harmonic to $A$, and it is directly related to the spectral amplification $\eta$ through the expression $Q=\sqrt{\eta}$ (see Ref. [2]). Notice that the above definition of the parameter $Q$ differs by a factor of 2 from the definition used in the theoretical approach in Ref. [20], and by a factor $A^{-1}$ from the one used in the numerical results appearing in the same reference. In the deterministic case, $D=0$, it has been shown that $Q$ goes through a maximum as the intensity of the high-frequency force is increased. This maximum is approximately localized around the value $r$ $=\sqrt{2 / 3}$ of the ratio of the amplitude of the second force to its frequency $[19,20]$. This behavior resembles that of SR, with the noise strength $D$ playing the role of the intensity of the high-frequency force.

When white noise is added, the situation is different. More precisely, the two main effects of increasing the value of the noise strength $D$ are that the value at the maximum of the resonance curve decreases and, at the same time, its location is shifted towards lower values of the high-frequency amplitude. Even more, for large enough values of $D$, the VR phenomenon completely disappears [20]. These effects can be qualitatively understood as a consequence of the fact that the white noise provides an input to the system with contributions to all the frequencies. On the one hand, the fraction corresponding to the high-frequency region advances the appearance of the maximum. On the other hand, the remaining contribution masks the high-frequency force, decreasing its relative importance [20]. To shed some more light on this question, an extension of the theoretical approach put forward in Ref. [20] to the case in which white noise is present would be desirable. This is the main aim of the following section.

\section{THEORETICAL APPROACH}

In this section, we will obtain analytical expressions for the parameter $Q$ based on three approximations of different nature. The first two ones are simply generalizations to the noisy problem of those carried out in the theoretical approach developed in the absence of noise [20]. The third approximation is specific to the noisy problem. For clarity in the exposition, as well as in the discussion of their validity conditions, we will present them separately.

\section{A. Derivation of the Effective Dynamics}

As a consequence of Eqs. (3) and (4), the time derivative of the probability density $P(x, t)$ diverges as $N$ in the limit $N \rightarrow \infty$. To avoid this divergence, it is convenient to extract the fast dependence from $x(t)$, and define the new stochastic process

$$
y(t)=x(t)-r \sin (N \Omega t+\varphi) .
$$

The Fourier components of $\langle x(t)\rangle_{\infty}$ and $\langle y(t)\rangle_{\infty}$ are related according to 


$$
Y_{n}=X_{n}+\frac{i r}{2}\left(e^{i \varphi} \delta_{n, N}-e^{-i \varphi} \delta_{n,-N}\right)
$$

where $Y_{n}$ is defined from Eq. (6), by replacing $\langle x(t)\rangle_{\infty}$ by $\langle y(t)\rangle_{\infty}$. Therefore, as $N \neq \pm 1$, we can substitute the coefficient $X_{1}$ by $Y_{1}$ in the definition of the parameter $Q$ [see Eq. (9)].

The advantage of using the process $y(t)$ instead of $x(t)$ becomes clear after writing the FPE for its probability density, which reads

$$
\frac{\partial}{\partial t} \mathcal{P}(y, t, \varphi)=\frac{\partial}{\partial y}\left[D \frac{\partial}{\partial y}+\mathcal{U}^{\prime}(y, t, \varphi)-A \cos (\Omega t)\right] \mathcal{P}(y, t, \varphi) .
$$

Here, we have introduced the new potential $\mathcal{U}(y, t, \varphi)$ $:=U[y+r \sin (N \Omega t+\varphi)]$, and the dependence of the functions on the phase $\varphi$ has been written explicitly. From Eq. (12) and the definition of $\mathcal{U}(y, t, \varphi)$ it follows that the time derivative of $\mathcal{P}(y, t, \varphi)$ is at most of order 1 as $N \rightarrow \infty$. Therefore, a large number of oscillations of the function $r \sin (N \Omega t+\varphi)$ appearing in the definition of $\mathcal{U}(y, t, \varphi)$ takes place before a significant change in $\mathcal{P}(y, t, \varphi)$ occurs. As a consequence, it is to be expected that, for $N \gg 1$, $\mathcal{P}(y, t, \varphi)$ is almost independent of the phase $\varphi$. If we define the phase average of an arbitrary function $f(y, t, \varphi)$ as

$$
\bar{f}(y, t)=\overline{f(y, t, \varphi)}:=\frac{1}{2 \pi} \int_{0}^{2 \pi} d \varphi f(y, t, \varphi)
$$

then, for $N \gg 1$ and any value of $\varphi \in[0,2 \pi]$, one can approximate $\mathcal{P}(y, t, \varphi) \approx \overline{\mathcal{P}}(y, t)$. Furthermore, the decoupling approximation $\overline{f(y, t, \varphi) \mathcal{P}(y, t, \varphi)} \approx \bar{f}(y, t) \overline{\mathcal{P}}(y, t)$ also holds. Carrying out the phase average in Eq. (12) and using the decoupling approximation, one obtains

$$
\frac{\partial}{\partial t} \overline{\mathcal{P}}(y, t)=\frac{\partial}{\partial y}\left[D \frac{\partial}{\partial y}+U_{e f f}^{\prime}(y)-A \cos (\Omega t)\right] \overline{\mathcal{P}}(y, t),
$$

where we have introduced the effective potential $U_{\text {eff }}(y)$ $:=\overline{\mathcal{U}(y, t, \varphi)}$, which is clearly time independent. This averaging procedure has been previously used in the study of thermal activation in bistable systems under the influence of a periodic force with moderate to large frequency [26]. It has also been used in the explanation of the enhancement observed in the tunnel splitting of a quantum bistable system when a very high frequency driving is applied [27].

It also follows from the above considerations that, for $N$ $\gg 1,\langle y(t)\rangle_{\infty} \approx\langle y(t)\rangle_{\infty}^{(e f f)}:=\int_{-\infty}^{\infty} d y y \overline{\mathcal{P}}_{\infty}(y, t)$, where $\overline{\mathcal{P}}_{\infty}(y, t)$ is the long time distribution corresponding to the FPE (14). In particular, within this approximation, the parameter $Q$ is given by

$$
Q=2 \frac{\left|Y_{1}^{(e f f)}\right|}{A},
$$

where $Y_{1}^{(e f f)}$ is obtained from Eq. (6) with $n=1$, by replacing $\langle x(t)\rangle_{\infty}$ by $\langle y(t)\rangle_{\infty}^{(e f f)}$.

The explicit calculation of $U_{e f f}(y)$ for the potential in Eq. (2) leads to

$$
U_{e f f}(y)=\frac{y^{4}}{4}-a(r) \frac{y^{2}}{2},
$$

where we have dropped an irrelevant constant and have introduced the quantity $a(r)=1-3 r^{2} / 2$. From this result, it is clear that the stability of this effective potential depends on the ratio $r$. More precisely, if $r<\sqrt{2 / 3}$ the potential is bistable, whereas if $r \geqslant \sqrt{2 / 3}$ it is monostable. Thus, an increase in $r$ leads to a decrease in the effective barrier height and, eventually, to its disappearance.

In summary, we have shown that, for $N \gg 1$, the time evolution of the original stochastic process $x(t)$ can be approximately described in terms of the dynamics of a Brownian particle moving in the effective potential $U_{\text {eff }}(y)$ and in the presence of noise and the monochromatic force $A \cos (\Omega t)$.

\section{B. Linear response theory}

Henceforth, following the approach in Ref. [20], we will assume that the amplitude $A$ is small enough, so that linear response theory (LRT) provides a good description of the effective dynamics obtained in the preceding section. An extensive study of the validity conditions of LRT can be found in Refs. [28-30]. Within LRT the parameter $Q$ in Eq. (15) is given [2] by

$$
Q=\left|\hat{\chi}_{e f f}(\Omega)\right|
$$

where

$$
\hat{\chi}_{e f f}(\Omega)=\int_{0}^{\infty} d t \chi_{e f f}(t) e^{-i \Omega t}
$$

is the value at $\omega=\Omega$ of the Fourier transform of the response function, $\chi_{e f f}(t)$, corresponding to an overdamped Brownian particle moving in the effective potential $U_{\text {eff }}(y)$. This response function obeys the well-known fluctuationdissipation theorem [31-33],

$$
\chi_{e f f}(t)=-\frac{\eta(t)}{D} \frac{d}{d t} K_{e f f}(t),
$$

where $\eta(t)$ is the Heaviside step function and $K_{\text {eff }}(t)$ $=\langle y(t) y(0)\rangle_{e q}^{(e f f)}$ is the equilibrium autocorrelation function of the effective system described by the FPE (14) in the absence of external driving. Thus, the stochastic process $y(t)$ appearing in the definition of $K_{\text {eff }}(t)$ is a solution of the SDE

$$
\dot{y}(t)=-U_{e f f}^{\prime}[y(t)]+\xi(t) .
$$

Use of Eq. (19) in Eq. (18) yields 


$$
\begin{gathered}
\operatorname{Re}\left[\hat{\chi}_{e f f}(\Omega)\right]=-\frac{\Omega}{D}\left[\int_{0}^{\infty} d t K_{e f f}(t) \sin (\Omega t)-\frac{K_{e f f}(0)}{\Omega}\right], \\
\operatorname{Im}\left[\hat{\chi}_{e f f}(\Omega)\right]=-\frac{\Omega}{D} \int_{0}^{\infty} d t K_{e f f}(t) \cos (\Omega t) .
\end{gathered}
$$

These two equations, together with Eq. (17), allow us to express $Q^{(L R T)}$ in terms of the equilibrium autocorrelation function $K_{\text {eff }}(t)$.

For the nonlinear potential in Eq. (16), explicit expressions for $K_{\text {eff }}(t)$ are unknown, so that this correlation function must be evaluated either numerically or by resorting to suitable approximations. Before applying to our problem approximate techniques discussed in the literature, it is convenient to reduce the SDE (20) to a more standard form. In order to do so, let us rescale the coordinate and time, and define the new stochastic process $\tilde{y}(\tilde{t})=|a(r)|^{-1 / 2} y\left[|a(r)|^{-1} \tilde{t}\right]$ (for $r \neq \sqrt{2 / 3}$ ). Then, from Eq. (20), it is easy to verify that $\tilde{y}(\widetilde{t})$ fulfills the SDE

$$
\dot{\tilde{y}}(\widetilde{t})=-\tilde{U}^{\prime}[\widetilde{y}(\widetilde{t})]+\widetilde{\xi}(\widetilde{t}),
$$

where we have introduced the rescaled potential

$$
\widetilde{U}(\widetilde{y})=\frac{\tilde{y}^{4}}{4}+\operatorname{sgn}[a(r)] \frac{\tilde{y}^{2}}{2},
$$

and the rescaled Gaussian white noise $\tilde{\xi}(\widetilde{t})$ with zero average and autocorrelation function $\left\langle\tilde{\xi}(\tilde{t}) \tilde{\xi}\left(\tilde{t}^{\prime}\right)\right\rangle=2|a(r)|^{-2} D \delta\left(\tilde{t}-\tilde{t}^{\prime}\right)$. From these considerations, it is straightforward to prove that the equilibrium autocorrelation function of the original process, $K_{\text {eff }}(t, D)$, and that of the rescaled one, $\widetilde{K}(\widetilde{t}, \widetilde{D})$, are related by

$$
K_{\text {eff }}(t, D)=|a(r)| \widehat{K}\left(|a(r)| t, \frac{D}{|a(r)|^{2}}\right) .
$$

Analogously, Eqs. (21), (22), and (25) lead to the following relation between the Fourier transform of the response function of the original process, $\left|\hat{\chi}_{e f f}(\Omega, D)\right|$, and that of the rescaled one, $|\hat{\bar{\chi}}(\widetilde{\Omega}, \widetilde{D})|$,

$$
\left|\hat{\chi}_{e f f}(\Omega, D)\right|=\frac{1}{|a(r)|}\left|\hat{\bar{\chi}}\left(\frac{\Omega}{|a(r)|}, \frac{D}{|a(r)|^{2}}\right)\right| .
$$

\section{Weak noise approximation}

The expressions (25) and (26) allow us to evaluate $K_{\text {eff }}(t, D)$ and $\left|\hat{\chi}_{\text {eff }}(\Omega, D)\right|$ from the rescaled functions $\widetilde{K}(\widetilde{t}, \widetilde{D)}$ and $|\hat{\bar{\chi}}(\widetilde{\Omega}, \widetilde{D})|$. The asymptotic behaviors of these rescaled functions for small values of the noise strength $\widetilde{D}$ have been widely studied in the literature. To summarize the results, we will consider separately two cases.

First, if $r<\sqrt{2 / 3}$, then $\widetilde{U}(\tilde{y})=\widetilde{y}^{4} / 4-\widetilde{y}^{2} / 2$ is the archetypal symmetric quartic double-well potential expressed in dimensionless form. For this bistable potential, we can use the two-mode approximation [34]. This approximation is based on the existence of a clear-cut separation between the time scales associated to interwell and intrawell motions, and it is expected to be valid for small values of the noise strength $\widetilde{D}$. Within this approximation, $\widetilde{K}(\widetilde{t}, \widetilde{D})$ and $|\hat{\bar{\chi}}(\widetilde{\Omega}, \widetilde{D})|$ are given by

$$
\widetilde{K}(\widetilde{t}, \widetilde{D})=g_{1}(\widetilde{D}) \exp \left[-\lambda_{1}(\widetilde{D}) \tilde{t}\right]+g_{2}(\widetilde{D}) \exp [-\alpha \widetilde{t}],
$$

and

$$
\begin{aligned}
\mid \hat{\bar{\chi}} \widetilde{\Omega}, \widetilde{D}) \mid= & \frac{1}{\widetilde{D}}\left\{\frac{2 \alpha g_{1}(\widetilde{D}) g_{2}(\widetilde{D}) \lambda_{1}(\widetilde{D})\left[\alpha \lambda_{1}\left(\widetilde{D)}+\widetilde{\Omega^{2}}\right]\right.}{\left[\lambda_{1}^{2}(\widetilde{D})+\widetilde{\Omega^{2}}\right]\left(\alpha^{2}+\widetilde{\Omega^{2}}\right)}\right. \\
& \left.+\frac{g_{1}^{2}(\widetilde{D}) \lambda_{1}^{2}(\widetilde{D})}{\lambda_{1}^{2}(\widetilde{D})+\widetilde{\Omega^{2}}}+\frac{g_{2}^{2}(\widetilde{D}) \alpha^{2}}{\alpha^{2}+\widetilde{\Omega^{2}}}\right\}^{1 / 2} .
\end{aligned}
$$

In the above expressions, the parameter $\alpha=\widetilde{U}^{\prime \prime}( \pm 1)=2$ is the curvature at the minima of the potential $\widetilde{U}(\widetilde{y})$, and $\lambda_{1}(\widetilde{D})$ is the smallest nonvanishing eigenvalue of the Fokker-Planck operator corresponding to the SDE (23). In the steepestdescent approximation, this eigenvalue is given to leading order in $\widetilde{D}$ by

$$
\lambda_{1}(\widetilde{D}) \approx \frac{\sqrt{2}}{\pi} \exp \left(-\frac{1}{4 \widetilde{D}}\right) .
$$

The weights $g_{1}(\widetilde{D})$ and $g_{2}(\widetilde{D})$ read

$$
\begin{gathered}
g_{2}(\widetilde{D})=\frac{\left[\lambda_{1}(\widetilde{D})+1\right] m_{2}(\widetilde{D})-m_{4}(\widetilde{D})}{\lambda_{1}(\widetilde{D})-\alpha}, \\
g_{1}(\widetilde{D})=m_{2}\left(\widetilde{D)}-g_{2}(\widetilde{D})\right.
\end{gathered}
$$

where

$$
\begin{aligned}
m_{2 n}(\widetilde{D}) & =\frac{\int_{-\infty}^{\infty} d \widetilde{y} \widetilde{y}^{2 n} e^{-\widetilde{U}(\tilde{y}) / \widetilde{D}}}{\int_{-\infty}^{\infty} d \widetilde{y} e^{-\widetilde{U}(\tilde{y}) / \widetilde{D}}} \\
& =\left(2 \widetilde{D)^{n / 2}} \frac{\Gamma(n+1 / 2) D_{-n-1 / 2}\left[-(2 \widetilde{D})^{-1 / 2}\right]}{\Gamma(1 / 2) D_{-1 / 2}\left[-(2 \widetilde{D})^{-1 / 2}\right]}\right.
\end{aligned}
$$

are the nonvanishing moments of the equilibrium distribution. Using the asymptotic expansion of the parabolic cylinder functions for small values of $\widetilde{D}$, these weights can be approximated by $g_{1}(\widetilde{D}) \approx 1-(\alpha+1) \widetilde{D} / \alpha$ and $g_{2}(\widetilde{D}) \approx \widetilde{D} / \alpha$ to first order in $\widetilde{D}$.

Second, if $r>\sqrt{2 / 3}$, then $\widetilde{U}(\tilde{y})=\tilde{y}^{4} / 4+\tilde{y}^{2} / 2$ is a monostable potential. In this case, for small values of $\widetilde{D}$, the autocorrelation function $\widetilde{K}(\widetilde{t}, \widetilde{D})$ is given [35] by 


$$
\widetilde{K}(\widetilde{t}, \widetilde{D})=\widetilde{D} \exp \left[-\left|\Lambda_{1}(\widetilde{D})\right| \tilde{t}\right]
$$

with

$$
\Lambda_{1}(\widetilde{D})=-2+\left(1-6 \widetilde{D)^{1 / 2}} .\right.
$$

Consequently, the Fourier transform of the response function reads

$$
|\hat{\widetilde{\chi}}(\widetilde{\Omega}, \widetilde{D})|=\frac{\left|\Lambda_{1}(\widetilde{D})\right|}{\left[\Lambda_{1}^{2}\left(\widetilde{D)}+\widetilde{\Omega^{2}}\right]^{1 / 2}\right.} .
$$

To evaluate $K_{e f f}(t, D)$ and $\left|\hat{\chi}_{e f f}(\Omega, D)\right|$ one simply introduces either the expressions (27) and (28) or expressions (33) and (35) into Eqs. (25) and (26). Finally, within this approximation, the parameter $Q$ will be evaluated by replacing in Eq. (17) the result obtained for $\left|\hat{\chi}_{e f f}(\Omega, D)\right|$. It is important to emphasize that the approximate expressions (27), (28), (33), and (35) have been obtained under the assumption that $\widetilde{D}$ is a small parameter. Therefore, the resulting expressions for $K_{e f f}(t, D),\left|\hat{\chi}_{e f f}(\Omega, D)\right|$, and $Q$ are expected to be valid only for small values of $D|a(r)|^{-2}$. Taking into account that, for $D \neq 0$, the parameter $D|a(r)|^{-2}$ diverges at $r=\sqrt{2 / 3}$, then it is clear that, even for $D \ll 1$, there exists a region around $r$ $=\sqrt{2 / 3}$ in which the resulting expressions for $K_{\text {eff }}(t, D)$, $\left|\hat{\chi}_{e f f}(\Omega, D)\right|$, and $Q$ are not applicable. In particular, the expression for $Q$ is expected to provide a successful explanation of the VR phenomenon whenever its maximum takes place at a value of $r$ outside the above mentioned region.

From the above considerations, it is also possible to obtain an expression for the location of the maximum characterizing the VR phenomenon in the presence of white noise. According to the numerical results put forward in Ref. [20], this maximum is located at a value $r_{M}$ such that $r_{M}<\sqrt{2 / 3}$. Then, to evaluate the parameter $Q$ in the neighborhood of $r_{M}$, one has to consider Eq. (28) and follow the procedure described in the above paragraph. As the expression thus obtained is rather cumbersome, it is convenient to introduce a further approximation. More precisely, we will assume that, around $r_{M}$, the contribution due to the intrawell motion is negligible, so that just the second term in Eq. (28) with $g_{1}(\widetilde{D})=1$ is present. Therefore, the parameter $Q$ as a function of $r$ obtained from Eqs. (17) and (26) is given by

$$
Q(r)=\frac{a(r)}{D} \frac{\lambda_{1}\left[D / a^{2}(r)\right]}{\left\{\lambda_{1}^{2}\left[D / a^{2}(r)\right]+[\Omega / a(r)]^{2}\right\}^{1 / 2}}
$$

for $r<\sqrt{2 / 3}$. The location of the maximum, $r_{M}$, is readily determined from the equation $Q^{\prime}\left(r_{M}\right)=0$, where $Q^{\prime}(r)$ represents the derivative of $Q(r)$ with respect to $r$. Consequently, from Eq. (36) it follows that $r_{M}$ obeys the transcendental equation

$$
\lambda_{1}^{2}\left[\frac{D}{a^{2}\left(r_{M}\right)}\right]=2\left[\frac{\Omega}{a\left(r_{M}\right)}\right]^{2}\left[\frac{a^{2}\left(r_{M}\right)}{4 D}-1\right] .
$$

After making the replacements $D / a^{2}\left(r_{M}\right) \rightarrow D$ and $\Omega / a\left(r_{M}\right)$ $\rightarrow \Omega$ in the above equation, the resulting expression re- sembles, except for a factor of 2, the transcendental equation obtained within the two-state model to determine the noise intensity that maximizes the spectral amplification (see, e.g., Ref. [2] and references therein). The difference in the factor of 2 is due to the fact that, in that case, only the noise intensity is varied, whereas in our case, by varying $r$, we are simultaneously modifying the frequency and the noise intensity.

The transcendental equation (37) provides an explanation for the numerically observed behavior of the location of the maximum as a function of $D$ (see Sec. II). For a fixed value of $D$, the function $\lambda_{1}^{2}\left[D / a^{2}(r)\right]$ is a monotonically increasing function of $r$ in the interval $[0, \sqrt{2 / 3})$. Also, for fixed values of $D$ and $\Omega, 2[\Omega / a(r)]^{2}\left[a^{2}(r) /(4 D)-1\right]$ is a monotonically decreasing function of $r$ in the same interval. Thus, the existence of a solution of Eq. (37) in the interval $(0, \sqrt{2 / 3})$ requires the inequality $\lambda_{1}^{2}\left[D / a^{2}(0)\right]=\lambda_{1}^{2}(D)<2[\Omega$ / $a(0)]^{2}\left[a^{2}(0) /(4 D)-1\right]=2 \Omega^{2}[1 /(4 D)-1]$. Therefore, the same inequality is required for the existence of VR. If we now keep $r$ and $\Omega$ fixed and look at both sides of Eq. (37) as a function of $D$, we notice that $2[\Omega / a(r)]^{2}\left[a^{2}(r) /(4 D)-1\right]$ is a monotonically decreasing function of $D$, whereas $\lambda_{1}^{2}\left[D / a^{2}(r)\right]$ is a monotonically increasing function of $D$. We then conclude that the abscissa at the intersection point between these two functions, $r_{M}$, is shifted towards the left as $D$ increases. This is exactly the behavior observed numerically in Ref. [20]. Obviously, VR disappears for values of $D$ greater than the critical value $D_{c}$ for which $r_{M}=0$. This critical value fulfills the transcendental equation

$$
\lambda_{1}^{2}\left(D_{c}\right)=2 \Omega^{2}\left[\frac{1}{4 D_{c}}-1\right] .
$$

\section{COMPARISON WITH THE NUMERICAL SOLUTION}

In this section, we compare the results obtained for the parameter $Q$ by means of the numerical solution of the SDE (1), with the analytical expressions obtained in the preceding section. In order to evaluate numerically the parameter $Q$, a large enough number of stochastic trajectories, $x^{(k)}(t)$, has been generated by integrating the SDE (1) for every realization of the white noise $\xi(t)$, starting from a given initial condition $x_{0}$. The numerical solution of the SDE (1) has been carried out by using the algorithm put forward in Ref. [36] (see also the Appendix in Ref. [30]). After allowing for a relaxation transient stage, we evaluate the long time average by

$$
\langle x(t)\rangle_{\infty}=\frac{1}{M} \sum_{k=1}^{M} x^{(k)}(t),
$$

where $M$ is the number of stochastic trajectories considered. Then, the parameter $Q$ is obtained from Eqs. (9) and (6), with $n=1$, by numerical quadrature.

In Fig. 1, we depict the dependence of $Q$ vs. $r$. The following set of parameter values has been considered: $N=50$, $\varphi=0, \Omega=3.4 \times 10^{-2}, A=1.1 \times 10^{-2}, D=1.34 \times 10^{-2}$ in panel 



FIG. 1. Dependence of the parameter $Q$ vs $r$ corresponding to the noise strength values $D=1.34 \times 10^{-2}$ [panel (a)], $D=6.72$ $\times 10^{-2}$ [panel (b)], $D=1.34 \times 10^{-1}$ [panel (c)], and $D=4.03 \times 10^{-1}$ [panel (d)]. The rest of the parameter values are $N=50, \varphi=0, \Omega$ $=3.4 \times 10^{-2}$, and $A=1.1 \times 10^{-2}$. With circles we have plotted the results obtained from the numerical solution of the SDE (1) together with Eqs. (2) and (3). With solid lines we have depicted the analytical values of $Q$ provided by Eqs. (17) and (26), and either Eqs. (28)-(32), for $r<\sqrt{2 / 3}$, or Eqs. (35) and (34), for $r>\sqrt{2 / 3}$. The dotted lines indicate the location of the critical value $r=\sqrt{2 / 3}$, and the dashed lines the location of the maximum predicted by Eq. (37).

(a), $D=6.72 \times 10^{-2}$ in panel (b), $D=1.34 \times 10^{-1}$ in panel (c), and $D=4.03 \times 10^{-1}$ in panel (d). They correspond to four representative cases appearing in Fig. 6 in Ref. [20]. Note that the parameter values appearing in that reference must be rescaled in order to reduce the bistable potential used there to the standard dimesionless form in Eq. (2). With circles we have plotted the results obtained from the numerical solution of the SDE (1) together with Eqs. (2) and (3). With solid lines we have depicted the analytical values of $Q$ provided by Eqs. (17) and (26), and either Eqs. (28)-(32), for $r<\sqrt{2 / 3}$, or Eqs. (35) and (34), for $r>\sqrt{2 / 3}$. The location of the critical value $r=\sqrt{2 / 3}$ is indicated by the vertical dotted lines. In addition, in panels (a) and (b), we have depicted with vertical dashed lines the locations of the maxima predicted by Eq. (37). The critical value of the noise strength at which the VR phenomenon disappears, obtained from Eq. (38), is $D_{c}=1.154 \times 10^{-1}$. The noise strength values in panels (c) and (d) are greater than $D_{c}$, so it is to be expected that the VR phenomenon is not present in these cases, as it is confirmed numerically.

A glance at Fig. 1 reveals that the analytical expressions provide a good description of the main features of the VR phenomenon in the presence of white noise, at least if the ratio $r$ is far enough away from the critical value $r=\sqrt{2 / 3}$. In particular, the analytical results describe correctly the shift of the location of the maximum towards lower values of $r$, as well as the decrease of the value of $Q$ at the maximum, as the noise strength increases. It is important to emphasize that, as we mentioned in Sec. III C, the analytical results are not applicable in a neighborhood of the critical value $r=\sqrt{2 / 3}$, as a consequence of the divergence of the effective noise strength $D /[a(r)]^{2}$. The appearance of the vertical asymptote at this critical value makes this failure evident. Note that, in panel (a), the agreement between the numerical and analyti- cal results around the maximum gets worse. This is due to the proximity of the location of this maximum to the critical value $r=\sqrt{2 / 3}$.

\section{CONCLUSIONS}

We have studied the motion of a particle in a bistable potential in the presence of white noise and an external bichromatic force, within the context of the VR phenomenon. Analytical expressions for the parameter $Q$ which quantifies this phenomenon have been obtained based on three simplifying approximations of different nature. First, the exact dynamics of the system has been reduced to that of a Brownian particle moving in an effective potential and under the influence of white noise and one of the monochromatic forces. In order to make this approximation, we have assumed that the other monochromatic force is a strong, high-frequency field. Second, we have applied LRT to express the parameter $Q$ in terms of the equilibrium autocorrelation function associated to the effective dynamics in the absence of the external driving. Finally, we have obtained analytical expressions for $Q$ within the weak noise approximation. To do so, it has been necessary to consider two disjoint regions of values of the ratio $r$ separated by the critical value $r=\sqrt{2 / 3}$. We have also obtained a transcendental equation for the location of the maximum of $Q(r)$ which characterizes the VR phenomenon, as well as for the condition under which this phenomenon disappears. It is important to emphasize that our analytical approach allows us to predict the three main effects of additive noise on the VR phenomenon independent of numerical simulations. In particular, we have the following

Equation (37) predicts that the location of the maximum characterizing the VR phenomenon shifts to the left as the noise strength increases.

The analytical expression for $Q$ predicts the lowering of the maximum value of the resonance curve as the noise strength increases.

Finally, our theoretical results also predict that the VR phenomenon disappears for large enough values of the noise strength. Furthermore, we also give an expression [cf. Eq. (38)] for the highest value of $D$ beyond which the VR phenomenon disappears.

Comparison with the numerical solution of the original SDE shows that these analytical expressions provide a good quantitative description of the VR phenomenon, at least if it takes place outside a critical region around $r=\sqrt{2 / 3}$. For sufficiently large $D$ values, this limitation is not important, as the VR maximum is well outside this critical $r$ region. In the very small noise limit, the peak in $Q$ appears around $r$ $=\sqrt{2 / 3}$ and our approach becomes problematic. Nevertheless, in this limit the VR phenomenon is well described by the previously known deterministic treatment. With this work, we have attempted to provide a theoretical background to the numerical studies on the influence of additive noise on VR carried out in Ref. [20]. 


\section{ACKNOWLEDGMENTS}

We acknowledge the support of the Dirección General de Enseñanza Superior of Spain by Projects Nos. BFM2002-
03822 (J. C.-P.) and BFM2000-0967 (J. P. B.). We also want to thank Professor M. Morillo and Professor P. Hänggi for their critical reading of the manuscript and fruitful discussions.
[1] R. Benzi, A. Sutera, and A. Vulpiani, J. Phys. A 14, L453 (1981).

[2] L. Gammaitoni, P. Hänggi, P. Jung, and F. Marchesoni, Rev. Mod. Phys. 70, 223 (1998).

[3] V. Anishchenko, A. Neiman, F. Moss, and L. SchimanskyGeier, Phys. Usp. 42, 7 (1999).

[4] P. Hänggi, ChemPhysChem 3, 285 (2002).

[5] J. J. Collins, C. C. Chow, and T. T. Imhoff, Phys. Rev. E 52, R3321 (1995).

[6] J. J. Collins, C. C. Chow, A. C. Capela, and T. T. Imhoff, Phys. Rev. E 54, 5575 (1996).

[7] C. C. Chow, T. T. Imhoff, and J. J. Collins, Chaos 8, 616 (1998).

[8] S. Barbay, G. Giacomelli, and F. Marin, Phys. Rev. Lett. 85, 4652 (2000).

[9] I. Goychuk and P. Hänggi, Phys. Rev. E 61, 4272 (2000).

[10] P. Hänggi, P. Jung, C. Zerbe, and F. Moss, J. Stat. Phys. 70, 25 (1993).

[11] A. Neiman and L. Schimansky-Geier, Phys. Rev. Lett. 72, 2988 (1994).

[12] A. Neiman and W. Sung, Phys. Lett. A 223, 341 (1996).

[13] D. Nozaki, D. J. Mar, P. Grigg, and J. J. Collins, Phys. Rev. Lett. 82, 2402 (1999).

[14] V. Anishchenko, A. Neiman, and M. Safonova, J. Stat. Phys. 70, 183 (1993).

[15] S. Sinha, Physica A 270, 204 (1999).

[16] P. S. Landa and P. V. E. McClintock, J. Phys. A 33, L433 (2000).

[17] E. Ullner, A. Zaikin, R. Bascones, J. García-Ojalvo, and J. Kurths, Phys. Lett. A 312, 348 (2003).

[18] A. A. Zaikin, L. López, J. P. Baltanás, J. Kurths, and M. A. F.
Sanjuán, Phys. Rev. E 66, 011106 (2002).

[19] I. I. Blekhman and P. S. Landa, Int. J. Non-Linear Mech. 39, 421 (2004).

[20] J. P. Baltanás, L. López, I. I. Blechman, P. S. Landa, A. Zaikin, J. Kurths, and M. A. F. Sanjuán, Phys. Rev. E 67, 066119 (2003).

[21] L. Gammaitoni et al.,Phys. Rev. Lett. 82, 4574 (1999).

[22] M. Löcher et al.,Phys. Rev. E 62, 317 (2000).

[23] J. L. Lebowitz and P. G. Bergmann, Ann. Phys. (N.Y.) 1, 1 (1957).

[24] H. Risken, The Fokker-Planck Equation (Springer-Verlag, Berlin, 1984).

[25] P. Jung and P. Hänggi, Phys. Rev. A 44, 8032 (1991).

[26] P. Jung, Z. Phys. B: Condens. Matter 76, 521 (1989).

[27] F. Grossmann, P. Jung, T. Dittrich, and P. Hänggi, Z. Phys. B: Condens. Matter 84, 315 (1991).

[28] J. Casado-Pascual, J. Gómez-Ordóñez, M. Morillo, and P. Hänggi, Europhys. Lett. 58, 342 (2002).

[29] J. Casado-Pascual, J. Gómez-Ordóñez, M. Morillo, and P. Hänggi, Fluct. Noise Lett. 2, L127 (2002).

[30] J. Casado-Pascual, C. Denk, J. Gómez-Ordóñez, M. Morillo, and P. Hänggi, Phys. Rev. E 67, 036109 (2003).

[31] R. Kubo, J. Phys. Soc. Jpn. 12, 570 (1957).

[32] R. Kubo, Rep. Prog. Phys. 29, 255 (1966).

[33] P. Hänggi and H. Thomas, Phys. Rep. 88, 207 (1982).

[34] P. Jung and P. Hänggi, Z. Phys. B: Condens. Matter 90, 255 (1993).

[35] C. W. Gardiner, Handbook of Stochastic Methods, 2nd ed. (Springer-Verlag, Berlin, 1985).

[36] H. S. Greenside and E. Helfand, Bell Syst. Tech. J. 60, 1927 (1981). 